\title{
Neonatal Physiological Jaundice and Repertoire of Movements on the Second or Third Days of Life
}

Jolanta Witanowska ( $\nabla_{\text {jwitanowska@sum.edu.pl ) }}$

Medical University of Silesia in Katowice https://orcid.org/0000-0002-5547-214X

Iwona Doroniewicz

Institute of Physiotherapy and Health Science, Academy of Physical Education in Katowice

Alicja Affanasowicz

Institute of Physiotherapy and Health Science, Academy of Physical Education in Katowice

\section{Beata Jarecka}

Medical University of Silesia in Katowice

\section{Monika Bugdol}

Silesian University of Technology in Gliwice

\section{Daniel Ledwoń}

Silesian University of Technology in Gliwice

\section{Marta Danch-Wierzchowska}

Silesian University of Technology in Gliwice

\section{Małgorzata Matyja}

Institute of Physiotherapy and Health Science, Academy of Physical Education in Katowice Joanna Wojcieszczyk-Bacia

Medical Center, City Hospital in Piekary Śląskie

\section{Andrzej Myśliwiec}

Institute of Physiotherapy and Health Science, Academy of Physical Education in Katowice

\section{Research Article}

Keywords: infant, physiological jaundice, writhing movements, neurodevelopmental diagnosis of infants

Posted Date: June 11th, 2021

DOl: https://doi.org/10.21203/rs.3.rs-579799/v1

License: (9) This work is licensed under a Creative Commons Attribution 4.0 International License.

Read Full License 
1 Neonatal physiological jaundice and repertoire of movements on the second or third days of life

2

3

4 5

6

7

8

9

10

11

12

13

Jolanta Witanowska ${ }^{1}$, Iwona Doroniewicz ${ }^{2}$, Alicja Affanasowicz ${ }^{2}$, Beata Jarecka ${ }^{3}$, Monika Bugdol ${ }^{4}$, Daniel Ledwoń $^{4}$, Marta Danch-Wierzchowska ${ }^{4}$, Malgorzata Matyja ${ }^{2}$, Joanna Wojcieszczyk-Bacia ${ }^{5}$, Andrzej Myśliwiec $^{2}$

${ }^{1}$ Pediatric Nursing Department in Bytom, Medical University of Silesia in Katowice, Poland

${ }^{2}$ Institute of Physiotherapy and Health Science, Academy of Physical Education in Katowice, Poland

${ }^{3}$ Department of Pediatrics in Bytom, Medical University of Silesia in Katowice, Poland

${ }^{4}$ Faculty of Biomedical Engineering, Silesian University of Technology in Gliwice, Poland

${ }^{5}$ Medical Center, City Hospital in Piekary Śląskie, Poland

Corresponding author:

Jolanta Witanowska, PhD in medical sciences

Pediatric Nursing Department in Bytom

41-902 Bytom, ul. Batorego 15

jwitanowska@sum.edu.pl 
Physiological jaundice appears after the first day of life and has a mild form in most cases. High bilirubin levels can lead to jaundice of the basal ganglia and cause severe movement disorders in the form of extrapyramidal forms of cerebral palsy. The aim of the study was to assess the effect of bilirubin levels on the motor activity of infants and the relationship between bilirubin levels and selected sociometric and biometric characteristics.

The study included a group of 77 newborns of both sexes. Medical records were analyzed to determine their socio- and biometric characteristics. Bilirubin levels were evaluated using transcutaneous bilirubin (TcB) measurement with a Minolta JM-103 jaundice meter according to Kramer's method consisting in bilirubin measurements in different parts of the body. The head measurement was considered the most important for infants on the second or third day of life, and it was considered a reference for other variables. Analysis of neonatal activity was performed using video recording with a Sony camera with Full HD 1080p resolution (1920x1080, 60 fps). Each infant was assessed independently by three experts qualified in Prechtel's General Movements Assessment diagnosis.

No statistically significant differences in quantitative movement characteristics were observed between infants with normal and elevated bilirubin levels. The relationships were found between sociometric and biometric characteristics and bilirubin levels measured on the head. The analyses indicated that in the course of physiological jaundice, the bilirubin levels had no effect on their qualitative and quantitative movement parameters in the group of infants studied.

Keywords: infant, physiological jaundice, writhing movements, neurodevelopmental diagnosis of infants

\section{Introduction}

Physiological jaundice appears after 24 hours of life, with its highest bilirubin levels reported between the 3rd and 5th days of life. In most cases, it has a mild course. High bilirubin levels can lead to jaundice of the basal ganglia. Therefore, bilirubin levels should be measured in every infant with jaundice to decide on treatment options. Visual assessment of jaundice is insufficient to evaluate its clinically significant severity. Therefore, transcutaneous bilirubin measurement is used, which does not require biochemical blood tests [1]. Neurological disorders due to bilirubin toxicity can appear with varying severity, from mild encephalopathy to kernicterus. At first, the symptoms are not very characteristic and include reluctance to suck, apathy, irritability, and decreased muscle tone. In the later stages, an increase in extensor muscle tone, extended head, hyperthermia, visual and auditory disorders, high-pitched cry, and seizures are observed [2]. Hyperbilirubinemia in infants may also manifest itself in later stages of life with delayed and disrupted cognitive, motor, and behavioral functions [3].

69 Based on the analysis of many reports published over the last two decades, non-invasive transcutaneous bilirubin $(\mathrm{TcB})$ meters are very useful as screening tools and provide a reliable estimate of total serum bilirubin (TSB). Furthermore, TcB measurement can help avoid potential errors associated with the visual assessment of jaundice and can be used as a screening device to detect jaundice and reduce the number of skin punctures [4]. 


\section{Study aim}

The aim of the study was to analyze the effect of bilirubin levels on motor activity of infants in terms of quality of motor patterns and selected kinematic parameters and to determine which independent variables, i.e. infant sex, place of residence, mother's and father's education, parents' professional activity, pregnancy sequence, birth weight, feeding method, type of delivery, rh incompatibility, and Apgar score determine bilirubin levels measured through the skin on the infant's head in the frontal region.

\section{Material and Method}

The study involved a group of 77 infants of both sexes (36 girls and 41 boys) born mostly ( $n=49$ ) by normal delivery, with a mean birth weight of $3404.68 \mathrm{~g}$, body length of $55.68 \mathrm{~cm}$, head circumference of 33.94 $\mathrm{cm}$, chest circumference of $33.93 \mathrm{~cm}$, and Apgar score of 8-10 points on the second or third day of life. The mean hourly age of the examination was 43.34 hours after birth.

The research procedure involved a retrospective analysis of medical records of infants hospitalized in the neonatal ward of the Piekary Medical Centre (Piekarskie Centrum Medyczne Sp. z o.o.) at St. Luke City Hospital in Piekary Śląskie. Data were collected from medical records of infants born during the examination conducted in 2019 and 2020. The transcutaneous bilirubin screening protocol involved the measurement of TcB with a Minolta JM-103 jaundice meter. Transcutaneous bilirubin levels were measured in regions according to the Kramer scheme [2]. Areas of skin with nevi, bruising, or any abnormality present were omitted as recommended by the device manufacturer. According to the information provided by the manufacturer of the Minolta JM-103, the device measures transcutaneous bilirubin levels ranging from 0 to $25 \mathrm{mg} / \mathrm{dl}$, with an accuracy of $1.5 \mathrm{mg} / \mathrm{dl}$. In the examinations according to the Kramer protocol, the following ranges of bilirubin were adopted as normal: $4-8 \mathrm{mg} \%$ on the head, $5-12 \mathrm{mg} \%$ on the chest, $8-16 \mathrm{mg} \%$ on the abdomen, $11-18 \mathrm{mg} \%$ on the limbs, and more than $18 \mathrm{mg} \%$ on the hands and feet [5]. It was decided that motor activity and selected biometric characteristics would be assessed in relation to $\mathrm{TcB}$ results in the head area. This procedure was justified by the fact that the infants were examined on the 2nd or 3rd day of life and jaundice appeared first on the head and then on the chest, abdomen, and hands and feet.

The analysis of movement patterns was based on video recordings obtained with a Sony camera with a Full HD 1080 resolution (1920x1080, $60 \mathrm{fps}$ ) based on the assumptions of the OSESEC project (Objective System for Evaluation and Support of Early Childhood). Each recording was obtained in a time frame of 10 minutes or less until the onset of the prolonged baby crying. During the recording, the infants were wearing only their diapers and placed in a supine position in a portable hospital cot. Recordings were made after the infant's feeding, with adjustments made to the infant's daily rhythm. Each infant was independently evaluated by three experts and classified into one of two categories of writhing movements (normal or poor repertoire) which are the earliest type of global movements described by Prechtel [6][7]. Writhing movements (WMs) are movements with amplitude and velocity from small to moderate [8]. These movements can last for several seconds, usually involving the entire infant's body. Complex sequences of extension and flexion movements with rotations along the long axes of the limbs were observed. Smoothness, diversity of amplitude and sequence, and complexity indicating association with rotation in movement along the long axis of the limb were assessed. The normal movements criterion meant at least three complete writhing movements recorded during observation. The poor repertoire group included infants whose observed movement components were monotonous and less complex 
than in the normal group [9]. The qualification was based on the unambiguous opinion of all three experts. In the case of disputable issues, the analysis was repeated (with open joint consultation). In the case of further disagreement, the participant was excluded from the observation group.

The next stage was the evaluation of kinematic parameters of movements. Due to the occurrence of factors interfering with the measurements, such as short crying, sleep, or changing the supine position of the infant to lying on the side, it was necessary to choose a homogeneous part of the recording for each infant. The analysis included 1-minute sections of the video recording selected based on observations. The kinematic parameters of the infant's limb movements were determined from the changes in wrist and ankle locations on the recordings during writhing movements. An automatic point detection procedure was performed on individual frames of the video, using the OpenPose library [9]. The obtained locations of points after the preprocessing stage [10] were used to calculate the mean velocity, instantaneous velocities, and instantaneous accelerations in the analyzed part. The results were converted from the pixel domain of the image to real units based on the cot width manually determined in the image.

Statistical analyses were performed to assess the relationships between selected biometric and sociometric parameters and bilirubin levels, and between parameters describing the qualitative and quantitative physical activity and bilirubin levels. In qualitative terms, the relationships concerned normal movements and poor repertoire versus bilirubin levels, whereas in quantitative terms - kinematic parameters of movements versus bilirubin levels.

The examinations were conducted as part of the project "A system for supporting, objectivization and automation of the methods of prospective assessment of infant's psychomotor development in the first year of life". The research was approved by the University Bioethics Committee for Scientific Research at the Academy of Physical Education in Katowice No. 5/2018 as of 19 April 2018. All infants were included in the study after written informed consent was obtained from their guardians and the management of the medical facility where the research was carried out.

Data was entered into Microsoft Excel spreadsheets and analyzed using the R language in the RStudio environment. Statistical analysis was performed using Student's t-test and Mann-Whitney U test to compare quantitative variables in two groups: the Mann-Whitney $U$ test was computed in case one of the following assumptions was not fulfilled: distribution normality (Shapiro-Wilk test), variance homogeneity (F test), or group number equality (chi-squared test). For the comparison of quantitative variables in four groups, the Kruskal-Wallis test was used due to the failure to meet the ANOVA assumptions in all analyzed cases. For posthoc analysis, the pairwise Wilcoxon test for unpaired data with Holm-Bonferroni correction was performed. The significance level was set at alpha $=0.05$. In the case of statistical significance of a test, the effect size was evaluated and only the effects larger or equal to moderate were reported.

\section{Results}

In the study group of infants, bilirubin levels were measured transcutaneously according to the Kramer scheme. The results on individual body parts were as follows: $7.04 \mathrm{mg} \%$ on the head in the frontal region, 7.09 $\mathrm{mg} \%$ on the upper body, $6.09 \mathrm{mg} \%$ on the abdomen, $5.54 \mathrm{mg} \%$ on the limbs, and $2.75 \mathrm{mg} \%$ on the hands and feet. In further analyses, as described in the Material and Methods section, only the results of the head measurement were used. In the study group of newborns, bilirubin levels were measured transcutaneously on 
the head in the frontal region, and data were obtained on the mean bilirubin level, which was $7.04 \mathrm{mg} \%$. The lowest recorded level was $5.7 \mathrm{mg} \%$, whereas the highest was $13.9 \mathrm{mg} \%$ (normal range: $4-8 \mathrm{mg} \%$ ).

The experts assessing writhing movements classified 35 infants in the normal movement $(\mathrm{N})$ group and 39 infants in the poor repertoire (PR) group. In the group of infants studied, no pathological movements suggesting the development of cerebral palsy described as cramped-synchronized and chaotic movements were observed. The table below (Tab. 1) provides information on bilirubin levels in infants from the normal movement and poor repertoire groups.

There were no significant correlations between bilirubin levels and motor activity of the infants studied. No differences in infant's motor repertoire were found in the groups studied. A comparison of bilirubin levels in infants with normal movements and poor repertoire did not show a statistically significant difference. The kinematic parameters of upper and lower limb movements are shown in Table 2. No statistically significant differences were demonstrated in the mean velocity of upper and lower limb movements and acceleration of movements between groups of infants with normal and elevated bilirubin levels. A small variability of the determined parameters was observed, resulting from the variety of movement patterns presented by infants on individual recordings.

The examinations revealed a relationship between some of the sociometric and biometric characteristics studied and bilirubin levels measured on the head. Median bilirubin was significantly higher in infants whose mothers were economically active than in those whose mothers did not work. Infants with normal bilirubin levels were significantly more likely to be born in subsequent deliveries than those with bilirubin above normal. Furthermore, infants whose bilirubin levels were above normal were heavier than those whose bilirubin was normal. The later after birth the bilirubin level was measured (age in hours), the more often these infants had bilirubin levels above normal compared to those whose bilirubin was normal. The analysis of further characteristics such as infant's sex, rh incompatibility, place of residence, father's professional activity, parents' education, infant's health status according to Apgar score, feeding method, and type of delivery had no significant influence on transcutaneously measured bilirubin levels on the head in the course of physiological jaundice.

\section{Discussion}

Transcutaneous bilirubin measurements have been conducted for many years to analyze their usefulness for screening neonatal physiological jaundice, thus reducing the need for invasive blood sampling for testing [11] [12] [13]. Most researchers have emphasized that TcB measurement is the best way to avoid unnecessary blood sampling, thus reducing stress on infants and their parents. This procedure also reduces the cost of medical care and, importantly, carries no risk to the health and life of the infants tested [14][15][16]. Furthermore, given that early discharge of newborns to home is now a common practice, transcutaneous bilirubinometry becomes a screening test that can be performed during home visits. The evaluation of bilirubin levels in infants is important as its concentration changes during the subsequent days of life and may not reach its maximum levels until the day of discharge. However, infants are at risk for significant neonatal hyperbilirubinemia. Visual assessment of skin or sclera color is not sufficiently accurate [11]. Maisels et al. took a similar position and recommended TcB 
measurements as a first-line screening tool to determine whether TSB should be measured [17]. Furthermore, Romagnoli et al. reported that the highest sensitivity of TcB measurements is achieved after 60 hours of infant

195

196

197

198

199

200

201

202

203

204

205

206

207

208 life [18]. In our study, transcutaneous bilirubin measurement of infants was conducted between 25 and 72 hours of their life.

Afanetti $\mathrm{M}$ et al. demonstrated that transcutaneous bilirubinometry is a safe and cost-effective screening method for severe hyperbilirubinemia in preterm and full-term infants of diverse ethnicity [18].

In our study, the highest bilirubin level was $13.9 \mathrm{mg} \%$ (normal values: $4-8 \mathrm{mg} \%$ ). It can be assumed that slightly elevated bilirubin does not differentiate infants in terms of motor activity (global movements). A peak serum bilirubin level (PSB) that is a predictor of abnormal psychomotor development in infants is greater than $\mathrm{PSB} \geq 22 \mathrm{mg} / \%$ [19]. In the available literature, there are no papers indicating motor disorders in infants with slightly elevated bilirubin levels. The disorders described include bilirubin-induced neurologic dysfunction (BIND) in infants with moderate hyperbilirubinemia who presented with an impaired sucking reflex, numbness, sleeplessness, and hypotonia [20],[21] and acute bilirubin encephalopathy (ABE) in infants with severe hyperbilirubinemia, who developed symptoms similar to BIND in the first days, then progressing to coma, highpitched cry, and hypertonia [23], and, as observed in clinical practice, reduced activity and some lethargy of infants with slightly elevated bilirubin levels. Both qualitative analysis of motor patterns (writhing movements) and quantitative analysis (evaluation of kinematic parameters) did not show statistically significant differences between infants with normal and elevated bilirubin levels.

Experts selected infants with normal movements and poor repertoire. However, the different motor levels did not show any associations with bilirubin levels.

The proposed methodology for the evaluation of kinematic parameters of limb movements allowed for the analysis of differences in velocity and acceleration between the group of infants with elevated bilirubin and its normal levels. The analysis showed no significant differences between these parameters. Slightly exceeding bilirubin levels does not significantly affect the kinematic parameters of the infant's spontaneous motor activity. The accuracy of the measurement method used in our study and the impact of the acquisition conditions limit the opportunities for observing possible small changes in the velocity in the case of neonatal physiological jaundice.

\section{Conclusion}

The analysis of the research material collected in the present study leads to the following conclusions:

1. Bilirubin levels in the course of physiological jaundice did not affect the quality of motor repertoire in the group of infants studied.

2. Higher birth weight was reported in infants whose bilirubin levels measured on the head were above normal.

3. Further research focused on the qualitative and quantitative motor analysis of infants with moderately and severely elevated bilirubin levels should be conducted. 


\section{References}

[1] Van Den Esker-Jonker B, Den Boer L, Pepping RMC, Bekhof J (2016) Transcutaneous bilirubinometry in jaundiced neonates: A randomized controlled trial. Pediatrics 138 (6): e20162414. doi: 10.1542/peds.20162414

[2] Wasiluk A, Polewko A, Ozimirski A (2012) Współczesna diagnostyka i leczenie żółtaczek u noworodków i niemowląt. Diagnostyka Lab 48 (4): 405-411.

[3] Nagar G, Vandermeer B, Campbell S, Kumar M (2013) Reliability of transcutaneous bilirubin devices in preterm infants: A systematic review. Pediatrics 132 (5): 871-881.

[4] Wusthoff CJ, Loe IM (2015) Impact of bilirubin-induced neurologic dysfunction on neurodevelopmental outcomes. Seminars in Fetal and Neonatal Medicine 20 (1): 52-57. DOI: 10.1016/j.siny.2014.12.003

[5] Kramer LI (1969) Advancement of Dermal Icterus in the Jaundiced Newborn. Am. J. Dis. Child. 118 (3): 118: 454-458.

[6] Carceller-Blanchard A, Cousineau J, Delvin EE (2009) Point of care testing: Transcutaneous bilirubinometry in neonates. Clinical Biochemistry 42 (3): 143-149.

[7] Einspieler C, Prechtl HFR, Ferrari F, Cioni G, Bos AF (1997) The qualitative assessment of general movements in preterm, term and young infants - Review of the methodology, Early Human Development 50 (1): 47-60. doi: 10.1016/s0378-3782(97)00092-3.

[8] Einspieler C, Prechtl HFR (2005) Prechtl's assessment of general movements: A diagnostic tool for the functional assessment of the young nervous system. Mental Retardation and Developmental Disabilities Research Reviews 11 (1): 61-67.

[9] Cao Z, Hidalgo G, Simon T, Wei SE, Sheikh Y (2021) OpenPose: Realtime Multi-Person 2D Pose Estimation Using Part Affinity Fields. IEEE Trans. Pattern Anal. Mach. Intell. 43 (1): 172-186 doi: 10.1109/TPAMI.2019.2929257.

[10] Doroniewicz I, Ledwoń, DJ, Affanasowicz A, Kieszczyńska K, Latos D, Matyja M, Mitas AW, Myśliwiec A. (2020) Writhing movement detection in newborns on the second and third day of life using pose-based feature machine learning classification. Sensors (Switzerland) $20 \quad$ (21): 5986. https://doi.org/10.3390/s20215986

[11] Rodríguez-Capote K, Kim K, Paes B, Turner D, Grey V (2009) Clinical implication of the difference between transcutaneous bilirubinometry and total serum bilirubin for the classification of newborns at risk of hyperbilirubinemia. Clin. Biochem. 42 (3): 176-179.

[12] Samiee-Zafarghandy S, Feberova J, Williams K, Yasseen AS, Perkins SL, Lemyre B (2014) Influence of skin colour on diagnostic accuracy of the jaundice meter JM 103 in newborns. Arch. Dis. Child. Fetal Neonatal Ed. 99 (6): F480-F484.

[13] Romagnoli C, Zecca E, Catenazzi P, Barone G, Zuppa AA (2012) Transcutaneous bilirubin measurement: Comparison of Respironics BiliCheck and JM-103 in a normal newborn population. Clin. Biochem. 45 (9): $659-662$.

[14] Akahira-Azuma M, Yonemoto N, Ganzorig B, Mori R, Hosokawa S, Matsushita T, Bavuusuren B, Shonkhuuz E. (2013) Validation of a transcutaneous bilirubin meter in Mongolian neonates: Comparison with total serum bilirubin. BMC Pediatr. 13 (1): 151 
[15] Morawiecka-Pietrzak M, Morawska H, Bursa J (2016) Evaluation of the correlation between transcutaneous measurement and concentration of bilirubin in the blood serum of a newborn", Pediatr. $\mathrm{i}$ Med. Rodz. 12 (3): 296-302

[16] Maisels MJ, Bhutani VK, Bogen D, Newman TB, Stark AR, Watchko JF (2009) Hyperbilirubinemia in the newborn infant $\geq 35$ weeks' gestation: An update with clarifications. Pediatrics 124 (4): 1193-1198

[17] Romagnoli C, Catenazzi P, Barone G, Giordano L, Riccardi R, Zuppa AA, Zecca1 E. (2013) BiliCheck vs JM-103 in identifying neonates not at risk of hyperbilirubinaemia. Ital. J. Pediatr. 39 (1): 46. doi: 10.1186/1824-7288-39-46

[18] Afanetti M, Eleni dit Trolli S, Yousef N, Jrad I, Mokhtari M (2014) Transcutaneous bilirubinometry is not influenced by term or skin color in neonates. Early Hum. Dev. 90 (8): 417-420.

[19] Arun Babu T, Bhat BV, Joseph NM (2012) Association between peak serum bilirubin and neurodevelopmental outcomes in term babies with hyperbilirubinemia. Indian J. Pediatr. 79 (2): 202-6. doi: 10.1007/s12098-011-0501-2

[20] Johnson L, Bhutani VK (2011) The Clinical Syndrome of Bilirubin-Induced Neurologic Dysfunction. Seminars in Perinatology 35 (3): 101-13. doi: 10.1053/j.semperi.2011.02.003.

[21] Bhutani VK, Johnson-Hamerman L (2015) The clinical syndrome of bilirubin-induced neurologic dysfunction. Seminars in Fetal and Neonatal Medicine 20 (1): 6-13. doi: 10.1016/j.siny.2014.12.008.

[22] Rose J, Vassar R (2015) Movement disorders due to bilirubin toxicity. Seminars in Fetal and Neonatal Medicine 20 (1): 20-25. doi: 10.1016/j.siny.2014.11.002. induced encephalopathy. Iran J Child Neurol 4 (1): 20-25. doi: 10.1016/j.siny.2014.11.002. 


\section{Supplementary Files}

This is a list of supplementary files associated with this preprint. Click to download.

- Table1.pdf

- Table2.pdf 